\title{
Bifocal Matching Using Multiple Geometrical Solutions
}

\author{
Miguel Carrasco ${ }^{1}$ and Domingo Mery ${ }^{2}$ \\ 1 Escuela de Informática y Telecomunicaciones \\ Universidad Diego Portales \\ Ejército 441, Santiago de Chile \\ miguel.carrasco@mail.udp.cl \\ 2 Departamento de Ciencia de la Computación \\ Pontificia Universidad Católica de Chile \\ Av. Vicuña Mackenna 4860(143), Santiago de Chile \\ dmery@ing.puc.cl
}

\begin{abstract}
Determining point-to-point correspondence in multiple images is a complex problem because of the multiple geometric and photometric transformations and/or occlusions that the same point can undergo in corresponding images. This paper presents a method of pointto-point correspondence analysis based on the combination of two techniques: (1) correspondence analysis through similarity of invariant features, and (2) combination of multiple partial solutions through bifocal geometry. This method is quite novel because it allows the determination of point-to-point geometric correspondence by means of the intersection of multiple partial solutions that are weighted through the MLESAC algorithm. The main advantage of our method is the extension of the algorithms based on the correspondence of invariant descriptors, generalizing the problem of correspondence to a geometric model in multiple views. In the sequences used we got an F-score $=97 \%$ at a distance of less than 1 pixel. These results show the effectiveness of the method and potentially can be used in a wide range of applications.
\end{abstract}

Keywords: computer vision, multiple view geometry, correspondence problem,tracking.

\section{Introduction}

The point-to-point correspondence analysis between two images made of the same scene is a very relevant problem in the computer vision community. Problems such as 3D reconstruction, robotic navigation, tracking in multiple views, estimation of transformation matrices and homographies, among others, are some of the applications that require the precise determination of correspondences. Correspondence analysis consists basically in determining a set of points in an image such that they are identified as the same in other images of the same scene. This situation is described clearly in Fig[1, which shows a possible corresponding point in three images of the same object. Different approaches for 


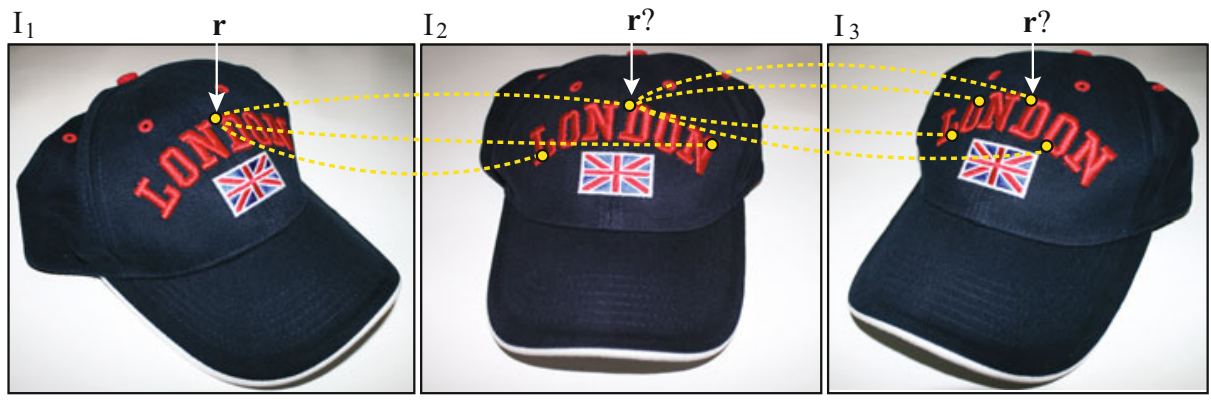

Fig. 1. General problem of correspondences in multiple views. Main objective: determining the correspondence of point $\mathbf{r}$ of image $\mathbf{I}_{1}$ in corresponding images $\mathbf{I}_{2}$ e $\mathbf{I}_{3}$.

solving the correspondence matching have been developed. Some of them are, for example, methods based on the analysis of invariant descriptors [311], estimation of affine transformations, homographies and estimation of perspective transformations 48, epipolar geometry analysis [1419, and methods based on optical flow [1]. In general, all these methods differ in the type of motion of the objects contained in a video sequence.

In spite of the large number of methods designed to solve this problem, the correspondence of images with very wide viewing angles has not been solved completely. This problem is commonly found when two or more independent cameras located at different positions and with wide perspective angles are used [5]. It is common to use techniques based on the analysis of invariant descriptors. Thanks to the invariance it is possible to solve and generalize the point-to-point correspondence problem by providing an extension of the methods based on stereo vision. That correspondence takes place as a function of the points of interest detected previously by some algorithm for detecting regions of interest [12]. However, when the point of interest does not correspond to a point detected by the current saliency techniques 10 18, how can we determine its corresponding pair in the other images? In this case, the previous methods do not ensure finding a correct correspondence because they are designed to maximize their performance only in the regions of interest detected by the method, and not necessarily in other regions. To avoid the problems of the correspondence methods mentioned previously, in this research we propose a new method to determine the pointto-point correspondence, particularly when the displacement angles are wide. Furthermore, since we use a geometric model that is independent of the objects, it is possible to determine the position of corresponding points in those views in which the point may be occluded. Graphically, we propose to solve the problem of Fig. 1. Given a point $\mathbf{r}$ in the image $\mathbf{I}_{1}$, the objective is to determine a corresponding point in the image $\mathbf{I}_{2}$.

In the following sections we detail our methodology for estimating the correspondence matrices in multiple views in uncalibrated sequences. The rest of the document is organized in the following sections: section 2 includes a description 
of the proposed method; section 3 includes the experiments and results; and finally, section 4 presents the conclusions and future work.

\section{Proposed Methodology}

In general, all the search methods for the fundamental matrix [9] have the purpose of finding the best model generated from a random set of pairs in correspondence through an error minimization process [72016]. This process can take place, for example, by means of a sampling consensus known as RANSAC [7], or the likelihood maximization in MLESAC by random sampling [16. Both methods, as well as the improvements proposed by Torrdoff and Murray [15], have been shown to be efficient methods for finding the fundamental matrices and perspectives in problems of computer vision. In the case of two views, the objective of the minimization process is to determine an epipolar single line in order to find an optimum epipole [14. However, these methods have been designed for problems in which there is a considerable number of erroneous correspondences, so the random search for hypotheses has the objective of determining the quality of each selected hypothesis and in that way reduce the selection of erroneous correspondences [16]. But what happens when there is a large number of correctly estimated correspondences? Is the best hypothesis the only solution that can be used? To answer these questions, below we present a new method for determining the point-to-point correspondence in two views in a geometric way.

\subsection{Correspondence in Two Views}

One of the most widely studied problems in computer vision is the geometric relation that exists between two corresponding images. A first step to solve this problem is to determine a set of point-to-point correspondences that estimate the geometric relations present in both images. In general, the problem of analysis in two views consists of how to determine the geometric relations of a $3 \mathrm{D}$ point and its projections on 2D planes. In what follows we will introduce the notation that relates the points in both images and the geometry that defines them. First, let $\mathbf{P}$ be a point in $3 \mathrm{D}$ space. In our example, point $\mathbf{P}$ is located in the upper corner of the $3 \mathrm{D}$ cube of Fig. 2. Second, let $\mathbf{C}_{1}$ and $\mathbf{C}_{2}$ be the optical centers of two cameras. For the following analysis, assume that we capture an image from the optical center $\mathbf{C}_{1}$, which generates image $\mathbf{I}_{1}$. Also, if we capture an image from the optical center $\mathbf{C}_{2}$, we generate image $\mathbf{I}_{2}$. According to this configuration, if we project a ray from center $\mathbf{C}_{1}$ to point $\mathbf{P}$, point $\mathbf{r}$ is generated on the $2 \mathrm{D}$ plane of image $\mathbf{I}_{2}$. Similarly, if we project a ray from center $\mathbf{C}_{2}$ to point $\mathbf{P}$, point $\mathbf{m}$ is generated, defined on the $2 \mathrm{D}$ plane of image $\mathbf{I}_{2}$. This relation implies that both rays intersect at a single point $\mathbf{P}$ defined in $3 \mathrm{D}$ space and its projections are on the $\mathbf{I}_{1}$ and $\mathbf{I}_{2}$ planes. In this way, points $\mathbf{r}$ and $\mathbf{m}$ correspond to a projection of point $\mathbf{P}$. In the ideal case both points are corresponding, since they were generated from a single point, in this case point $\mathbf{P}$. On the contrary, if we do not know the existence of point $\mathbf{P}$ we cannot assure that the correspondence is true. 


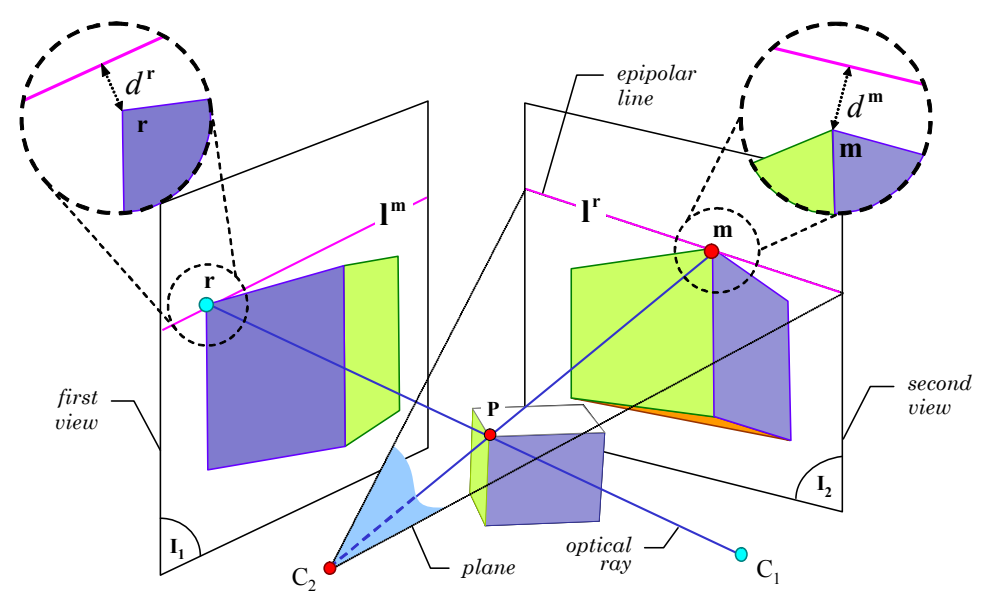

Fig. 2. General epipolar geometry of a $3 \mathrm{D}$ object and its projections

The latter situation is what normally occurs in point-to-point correspondence problems. In what follows we will denote by $\{\mathbf{r} \leftrightarrow \mathbf{m}\}$, when points $\mathbf{r}$ and $\mathbf{m}$ are corresponding, and as $\{\mathbf{r} \mapsto \mathbf{m}\}$ when the relation is hypothetical, i.e., we do not know if the relation is true or false and we want to find out.

A conventional way of proving the relation between points $\mathbf{r}$ and $\mathbf{m}$ is through the fundamental matrix $\mathbf{F}$ [914]. Formally, the fundamental matrix encapsulates the intrinsic geometry of two views, called epipolar geometry. For its determination it is necessary to know a minimum set of correspondences in both views. The main relation that establishes it is: given a pair of $\{\mathbf{r} \leftrightarrow \mathbf{m}\}$ correspondences, they always satisfy the following epipolar restriction:

$$
\mathbf{m}^{\top} \cdot \mathbf{F} \cdot \mathbf{r}=0
$$

Unfortunately, this relation is valid for all the points that are found at the intersection of the projection plane of the optical center $\mathbf{C}_{2}$ and the $\mathbf{I}_{2}$ plane. The line generated by that intersection is known as epipolar line 9 . Since point $\mathbf{r}$ belongs to the plane of the optical center $\mathbf{C}_{2}$, we say that the epipolar line in the second view $\mathbf{I}_{2}$ is correspondent with point $\mathbf{r}$ in the first view $\mathbf{I}_{1}$. According to this analysis, it is not possible to determine a biunivocal relation between points $\mathbf{r}$ and $\mathbf{m}$ using only an epipolar line. Various methods for estimating the fundamental matrix have been developed in recent years, e.g. 9]2. Regardless of the method for estimating the fundamental matrix, once it is determined it is possible to calculate the epipolar line described above, as shown in Fig. 2. Formally, let $\mathbf{l}^{\mathbf{r}}$ be the epipolar line of point $\mathbf{r}$ located in view $\mathbf{I}_{2}$, defined as $\mathbf{l}^{\mathbf{r}}=\mathbf{F} \cdot \mathbf{r}$. Let us assume that points $\mathbf{r}$ and $\mathbf{m}$ are corresponding. Therefore, they must be on epipolar lines $\mathbf{l}^{\mathbf{r}}$ and $\mathbf{l}^{\mathbf{m}}$ because they belong to the same plane. That is, $\mathbf{l}^{\mathbf{m}} \cdot \mathbf{r}=0$ and $\mathbf{l}^{\mathbf{r}} \cdot \mathbf{m}=0$. However, in practice the measurements of both views are not precise, and this implies that the epipolar lines do not necessarily 


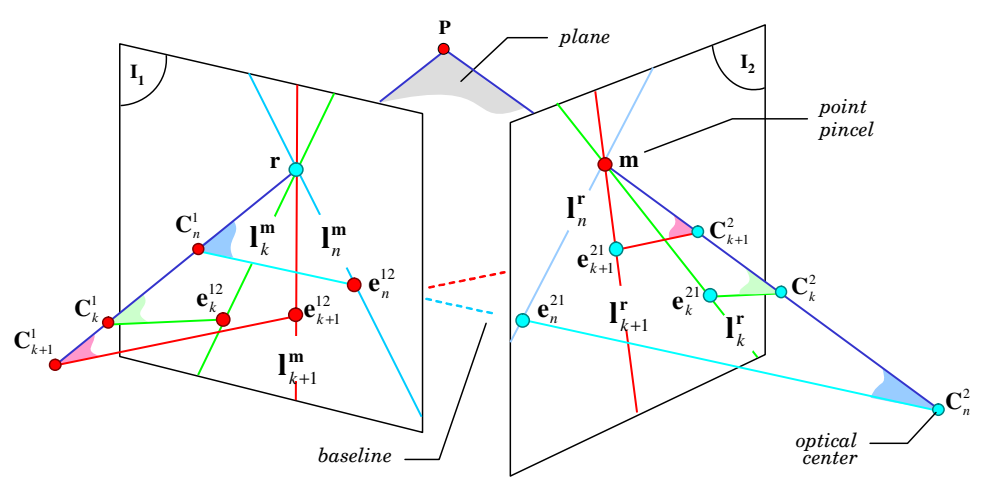

Fig. 3. Family of $n$ multiple epipolar lines in two views from multiple epipoles

intersect the points in correspondence. This error is reflected in the Euclidian distances $d^{\mathbf{r}}$ and $d^{\mathbf{m}}$ between the real point and the epipolar line, in which $d^{\mathbf{r}}>0$ and $d^{\mathbf{m}}>0$ (Fig. 2). Both distances should be minimal so that the projections are correct. To minimize that error it is necessary to determine a set of correspondences that minimize a reprojection error. Normally the correspondence selection methods use some distance measure or probabilistic value to carry out that minimization. In some cases the error is generated by optical distortions belonging to the lenses or by Gaussian noise present in the acquisition of the coordinates in correspondence.

An important point related to the estimation of the fundamental matrix is its dependence with respect to the set of correspondences used; i.e., for every set of correspondences a new fundamental matrix is determined. Even when the fundamental matrices are different, all of them remain valid provided $|\mathbf{F}|=0$. However, every fundamental matrix has associated with it a level of error due to the inaccuracies of the set of correspondences used. In spite of this error, the use of multiple fundamental matrices has two important advantages. (1) Every new fundamental matrix defines a new epipole position in the $\mathbf{I}_{1}$ and $\mathbf{I}_{2}$ planes. (2) The intersection of the epipole and the hypothetical point in correspondence $(\mathbf{r}$ or $\mathbf{m})$ generates a new epipolar line. Taking into account the two previous properties, let us assume that we choose $k$ sets in correspondence, where $k \in[1, \ldots, n]$ and $n$ is the maximum number of sets in correspondence. According to Fig. 3 the $\mathbf{e}_{k}^{12}$ and $\mathbf{e}_{k}^{21}$ epipoles are defined as the points of intersection between the baseline of the optical centers $\mathbf{C}_{k}^{1}$ and $\mathbf{C}_{k}^{2}$, and the $\mathbf{I}_{1}$ and $\mathbf{I}_{2}$ planes, respectively.

In this case, for the model proposed in Fig. 3 we assume that the position of point $\mathbf{P}$ is fixed. To illustrate the process in two views, we will assume that given a point $\mathbf{r}$ in the first view, there is a corresponding point in the second view. Since we do not know that correspondence, in our example we will assume that there are three hypothetical corresponding points, that we will call $\mathbf{m}, \mathbf{n}$, and p. As shown in Fig. 4, for the first set of correspondences the epipolar line $\mathbf{l}_{1}^{\mathbf{r}}$ intersects points $\mathbf{m}, \mathbf{n}$, and $\mathbf{p}$ in the second view $\mathbf{I}_{2}$. Therefore, let $\Theta$ be the set of hypothetical correspondences, where $\Theta=\{\{\mathbf{r} \mapsto \mathbf{m}\},\{\mathbf{r} \mapsto \mathbf{n}\},\{\mathbf{r} \mapsto \mathbf{p}\}\}$. Our objective is to determine a single correct pair of set $\Theta$; i.e., select the $\{\mathbf{r} \leftrightarrow \mathbf{m}\}$ 

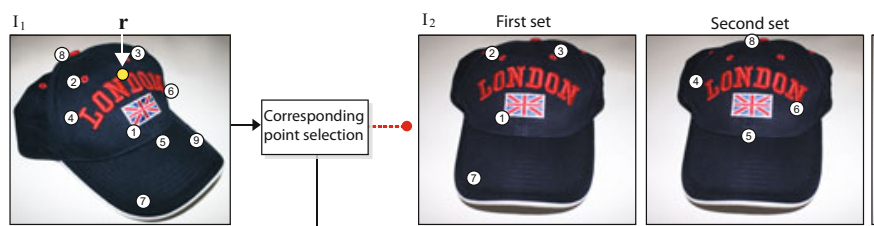

Third set
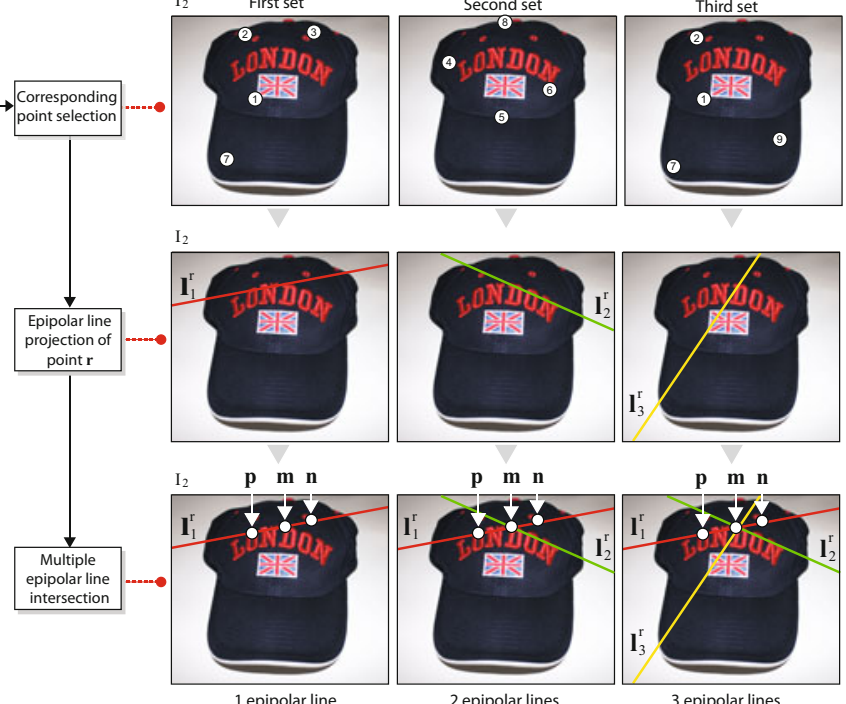

p $\mathbf{m ~ n}$ p $\mathbf{m} \mathbf{n}$

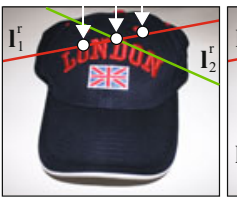

2 epipolar lines

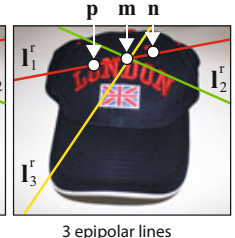

Fig. 4. New epipolar lines are created when a new set of corresponding points is created. In the example, line $\mathbf{l}_{1}^{\mathbf{r}}$ was obtained with set points $\{1,2,3,7\}$, line $\mathbf{l}_{2}^{\mathbf{r}}$ with set points $\{4,5,6,8\}$, and finally, line line $\mathbf{1}_{3}^{\mathbf{r}}$ with set points $\{1,2,7,9\}$.

pair and consequently discard the incorrect pairs. Based on the above discussion, if we intersect two epipolar lines $\mathbf{l}_{1}^{\mathbf{r}}$ and $\mathbf{l}_{2}^{\mathbf{r}}$, - both generated by two different subsets of correspondences- it is clearly seen that line $\mathbf{l}_{2}^{\mathbf{r}}$ is at a considerable distance from the correspondences $\mathbf{n}$ and $\mathbf{p}$. Similarly, a third epipolar line $\mathbf{l}_{3}^{\mathbf{r}}$ intersects the two previous ones at point $\mathbf{m}$ because the set of projected epipolar lines of point $\mathbf{r}$ intersect only one corresponding point in the second view, which in this case is point $\mathbf{m}$, generating an point pincel. That effect is repeated in both images, as shown by the model of Fig. 3 and Fig. 4 .

Theoretically, every new epipolar line improves the precision of corresponding point. However, in practice there is no single intersection point because of the uncalibrated nature of corresponding points used to formulate the geometric model, giving rise to an error in the estimation of the fundamental matrix. According to this analysis, one of the main problems in the estimation of the epipolar lines consists of determining their error level. Clearly, not all the epipolar lines have the same error, and for that reason we designed a method to determine the error associated with the Euclidian distance of each epipolar line. For the following analysis we will introduce the distance notation between the hypothetical point with respect to the epipolar line in the second view. Let $d_{k}^{\mathbf{m}}$ be the Euclidian distance between the $\mathbf{m}$-th point of the second view and the epipolar line $\mathbf{l}_{k}^{\mathbf{r}}$, where $\mathbf{r}$ is the $r$-th point of the first view. The distance $d_{k}^{\mathbf{m}}$ is defined as

$$
d_{k}^{\mathbf{m}}=\frac{\left|\mathbf{m}^{\top} \mathbf{F}_{k} \mathbf{r}\right|}{\sqrt{\left(\mathbf{F}_{k} \mathbf{r}\right)_{1}^{2}+\left(\mathbf{F}_{k} \mathbf{r}\right)_{2}^{2}}}
$$




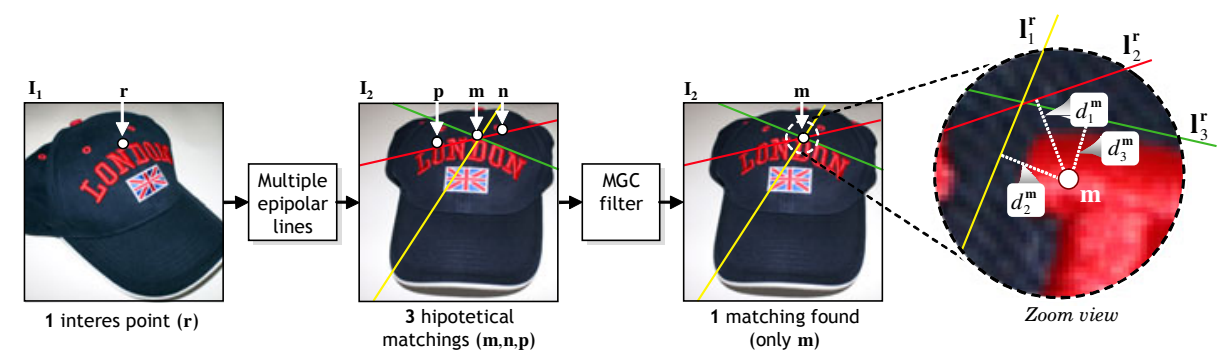

Fig. 5. The Multiple Geometric Correspondence (MGC) filter allows the determination of the point-to-point correspondence and distinguishing incorrect correspondences $\{\mathrm{p}, \mathrm{n}\}$

where $\left(\mathbf{F}_{k} \mathbf{r}\right)_{i}$ is the $i$-th component of vector $\mathbf{F}_{k} \mathbf{r}$. As mentioned earlier, our objective is to find the correspondence $\{\mathbf{r} \leftrightarrow \mathbf{m}\}$ of the set $\Theta$. However, we do not know the estimation error of each epipolar line. To make that estimation we will use the MLESAC algorithm, proposed by Torr and Zisserman 17. The objective of this process is to reestimate the Euclidian distances $d_{1}^{\mathbf{m}}, d_{2}^{\mathbf{m}}$ and $d_{3}^{\mathbf{m}}$ weighting the error of each epipolar line, a process that will be described below.

First we will introduce briefly some previous concepts of the MLESAC algorithm [17] to give greater clarity to the reader. MLESAC is a robust estimation algorithm to establish the point correspondences in multiple views, generalizing the RANSAC estimator [7. In our proposal MLESAC is an intermediate step in the error estimation process because the error estimated by MLESAC later allows weighting the individual error of each epipolar line. One of the main advantages of MLESAC is that it is designed considering that the error $P(e)$ is a mixture of Gaussians and uniform distributions, where $e$ is the error of the estimation of the fundamental matrix such that

$$
P(e)=\left(\gamma \frac{1}{\sqrt{2 \pi \sigma^{2}}} \exp \left(-\frac{e^{2}}{2 \sigma^{2}}\right)+(1-\gamma) \frac{1}{\nu}\right)
$$

where $\gamma$ is a mixing parameter, $\nu$ is an a priori constant that indicates the distribution of the data, and $\sigma$ is the standard deviation of the error in each coordinate. Parameters $\gamma$ and $\nu$ are not known, but they can be estimated by means of the EM [6] algorithm. In this way, the objective function is to minimize the log-likelihood of the error, which in our case is the distance $d_{k}^{\mathbf{m}}$ between a point and the epipolar line, and therefore

$$
-L_{k}=-\sum_{k}\left(\gamma\left(\frac{1}{\sqrt{2 \pi \sigma^{2}}}\right)^{n} \exp \left(-\frac{\left(d_{k}^{\mathbf{m}}\right)^{2}}{2 \sigma^{2}}\right)+(1-\gamma) \frac{1}{\nu}\right)
$$

We had previously mentioned that $\gamma$ and $\nu$ are not known. For completeness, we now indicate how they are estimated. Assuming that there are $k$ sets of correspondences, let $\eta_{k}$, where $\eta_{k}=1$ if the correspondence is correct, i.e., $d_{k}^{\mathbf{m}}=0$, and $\eta_{k}=0$ if the $k$ correspondence is incorrect. The EM algorithm considers that $\eta_{k}$ is an unknown value, and therefore it takes the following steps 
for its estimation: (1) it generates an initial value for $\gamma,(2)$ it estimates the $\eta_{k}$ value using the initial $\gamma$ estimation, and (3) it makes an estimation of $\gamma$ from the new estimated value $\eta_{k}$, and returns to step (2). The process is repeated until it converges. For this, let $p_{k}$ be the likelihood of distance $d_{k}^{\mathbf{m}}$ when it is an inlier, and $p_{o}$ the likelihood of distance $d_{k}^{\mathbf{m}}$ when it is an outlier. Consequently, given the initial value of $\gamma=\frac{1}{2}$, the probabilities $p_{k}$ and $p_{o}$ are estimated according to

$$
\begin{aligned}
& p_{k}=\gamma\left(\frac{1}{\sqrt{2 \pi \sigma^{2}}}\right)^{2} \exp \left(-\frac{d_{k}^{\mathbf{m}^{2}}}{2 \sigma^{2}}\right) \\
& p_{o}=(1-\gamma) \frac{1}{\nu}
\end{aligned}
$$

Once the probabilities $p_{k}$ and $p_{o}$ have been estimated from the initial value $\gamma$, the following step is to reestimate the $P\left(\eta_{k}=1 \mid \gamma\right)$ value according to

$$
P\left(\eta_{k}=1 \mid \gamma\right)=\frac{p_{k}}{p_{k}+p_{o}}
$$

and finally, in the phase called 'maximization' of step (3), the value $\gamma$ is reestimated according to the updated mixture of the probabilities $p_{k}$ and $p_{o}$,

$$
\gamma=\frac{1}{n} \sum_{k}\left(\frac{p_{k}}{p_{k}+p_{o}}\right)
$$

Normally three iterations are needed for the algorithm to converge. In this way the estimation of the log-likelihood of the $k$-th hypothesis of each epipolar line allows us to weight correctly the real distance $d_{k}^{\mathbf{m}}$. To make that estimation we will use the partial values of the log-likelihood $\left(L_{k}\right)$ and in that way we weight the distance $d_{k}^{\mathbf{m}}$ according to the following formulation:

$$
\tilde{d}_{k}^{\mathbf{m}}=d_{k}^{\mathbf{m}}\left(\frac{\left|\min \left(L_{k}\right)-L_{k}\right|+1}{\sum_{k}\left(L_{k}\right)}\right) .
$$

where $\tilde{d}_{k}^{\mathbf{m}}$ is a weighted distance that considers the error associated with each fundamental matrix. This procedure allows weighting and reestimating the distance of the epipolar lines according to the log-likelihood of the projection error with respect to the set of hypothetical points in the second view. The estimation of the error allows weighting correctly the distance $d_{k}^{\mathbf{m}}$, increasing or decreasing it according to the size of its error. Therefore, to determine a correspondence, we determine the distance with respect to the set $\Theta$. Finally, to identify the correspondence of point $\mathbf{r}$ the following relation must be satisfied:

$$
\tilde{d}_{k}^{\mathbf{m}}<\epsilon,
$$

where $\epsilon$ is a distance measured in pixels. The final result allows the determination of which points are corresponding and which, depending on a threshold level, must be discarded. Fig. 5 presents an example of how the error estimation discards points $\mathbf{n}$ and $\mathbf{p}$ from the set of correspondences $\Theta$. In particular, the 


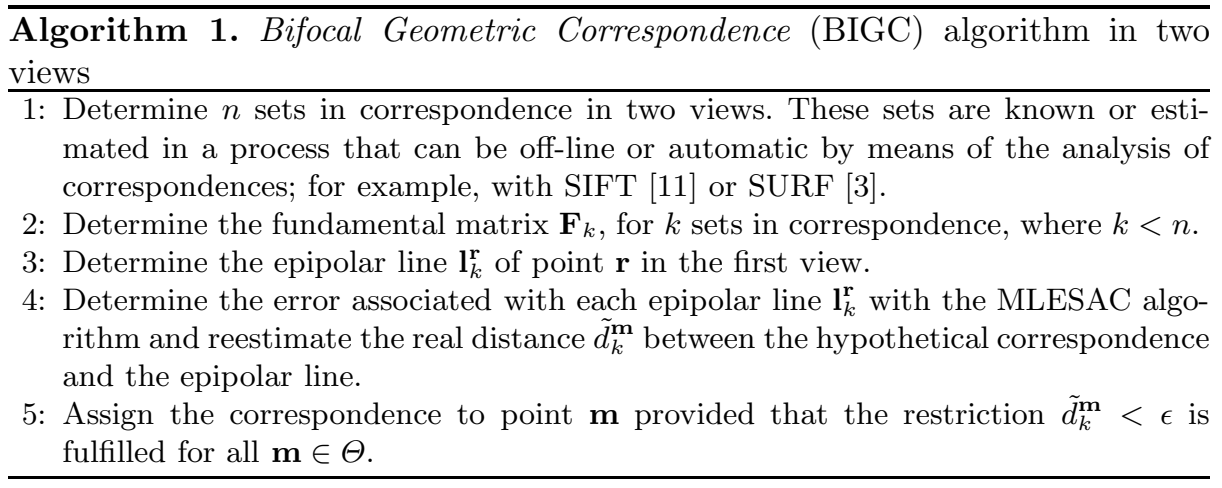

Multiple Geometric Correspondence MGC filter' block is in charge of reestimating the distances (Fig. 5). In the example, once point $\mathbf{m}$ is chosen, only the $\{\mathbf{r} \leftrightarrow \mathbf{m}\}$ combination is possible.

As shown in the previous steps, in spite of the errors existing in the estimation of the epipolar lines, their set allows the estimation of point-to-point correspondence. A complete description of the proposed methodology, which we call Bifocal Geometric Correspondence (BIGC), is presented in the 1Algorithm.

\section{$3 \quad$ Experimental Results}

This section presents the experimental results generated with sequences of uncalibrated images in two views. A set of 10 stereo images composed mainly of landscapes and walls, most of them supplied by the authors (Fig. 6) were used. In all the experiments we have considered two standard indicators 13: $r=\frac{\mathrm{TP}}{\mathrm{TP}+\mathrm{FN}}$ (recall) and $p=\frac{\mathrm{TP}}{\mathrm{TP}+\mathrm{FP}}$ (precision). TP is the number of true positives or correctly classified correspondences. FN is the number of false negatives or real correspondences not detected by our algorithm. FP is the number of false positives or correspondences classified incorrectly. These two indicators can be joined in a single measure $\mathrm{F}$-score $=\frac{2 \cdot p \cdot r}{p+r}$ [13]. Ideally, one can expect that $r=100 \%, p=100 \%$, and $\mathrm{F}$-score $=1$.

The first test set is composed of 10 pairs of images with a resolution of 1200 $\times 800$ pixels. The main existing geometric transformations are perspective, rotation, translation, and different degree scale (Fig. 6). This set consists of landscapes and walls in settings with natural lighting, showing a large number of regions in correspondence. According to the steps described in the 1 Algorithm, the first step consists of determining $n$ sets of corresponding pairs. This process was performed with the SURF 3 algorithm, from which we selected the best $k$ sets with the least projection error according to the MLESAC estimator. To evaluate the performance of the algorithm we determined 300 corresponding points in random positions within each pair of images in a process carried out off-line by means of the SURF algorithm. We then evaluated the ability of the algorithm to determine the correspondence by varying the $k \in[1, \ldots, 14]$ parameter and 
the $\epsilon \in[0, \ldots, 10]$ parameter. Note that in the latter parameter the values are rounded.

Below we present the results according to the variations of the $k$ and $\epsilon$ parameters. In the first case we analyze the influence of the $k$ parameter keeping distance $\epsilon$ fixed. As seen in Fig. 77, our best performance had an F-score $=0.97$ at a discretized distance $\epsilon=0$, using the intersection of three fundamental matrices $(k=3)$. It is interesting to mention that as the $\epsilon$ parameter increases, performance starts dropping. This indicates that the method is very precise in these kinds of images because there is a large number of correspondences. In the second case we analyze the influence of parameter $\epsilon$ keeping fixed the number of solutions $k$. According to the results obtained, we see a maximum performance at $k=3$. On the contrary, an increase of this value decreases the performance of the algorithm because the projection error increases.

Remember that in the analyzed sequence there is a large number of correspondences in spite of the geometric transformations present in them. Therefore, these results indicate that it is possible to use and estimate geometric models in two images with high precision at a subpixel resolution. According to the performance indicated in Fig. $7 \mathrm{a}$, after $k=4$ there is no improvement in the performance for $\epsilon>4$.
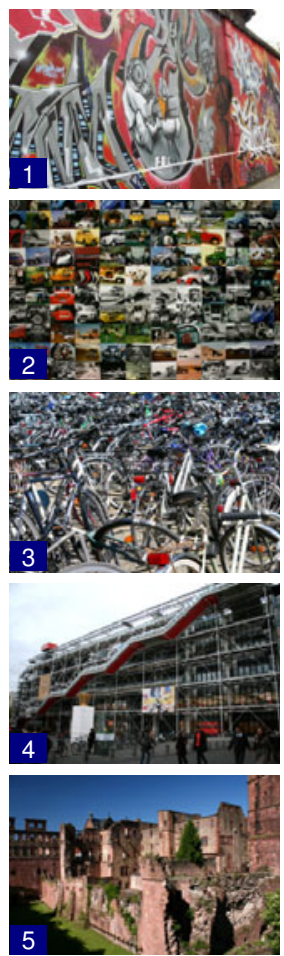
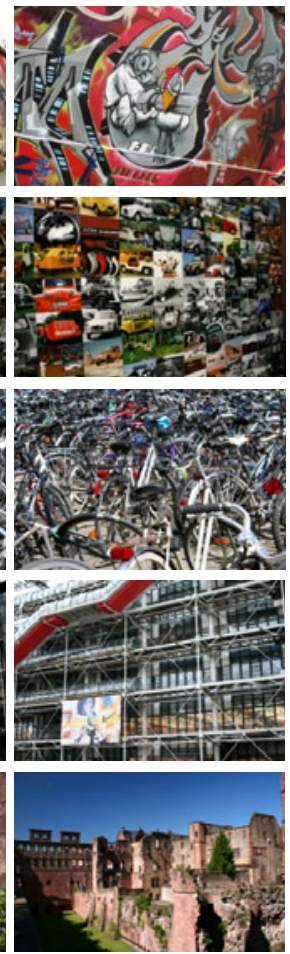
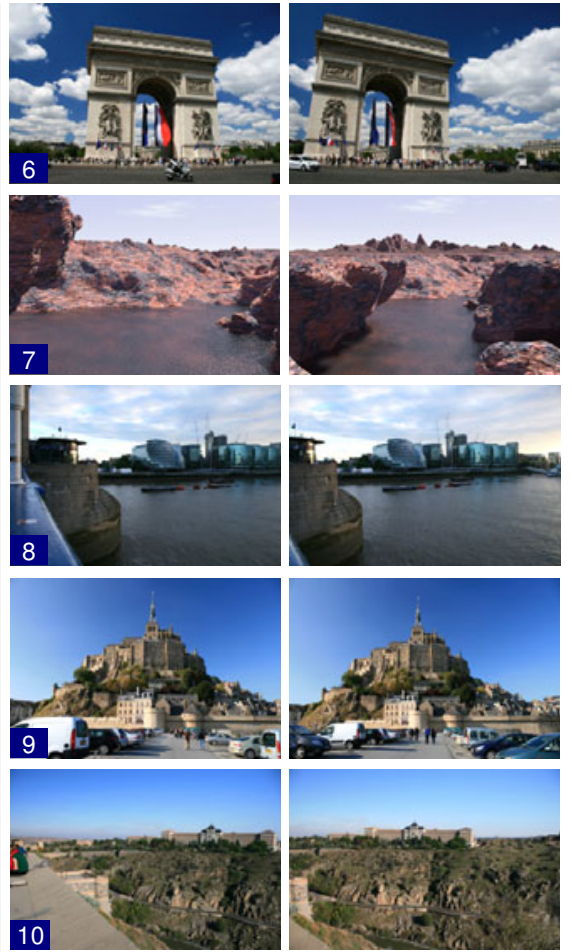

Fig. 6. Outdoor set of 10 stereo images 


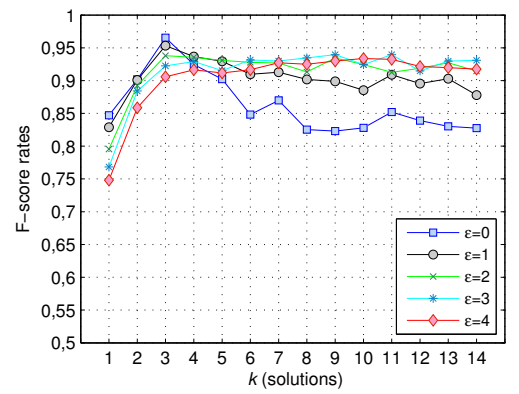

(a)

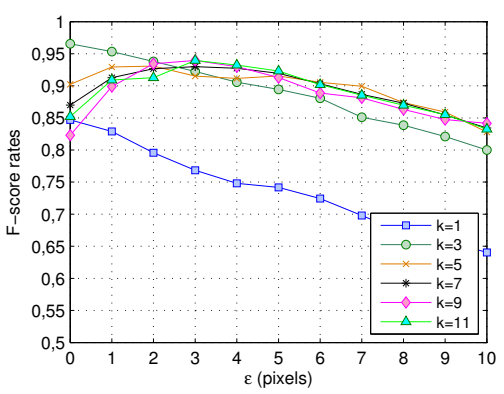

(b)

Fig. 7. Average performance of the set of outdoor images (a) Influence of the $k$ parameter as the maximum tolerance distance in pixels $\epsilon$ varies (b) Influence of distance $(\epsilon)$ on the detection of correspondences for different numbers of solutions $(k)$

\section{Conclusions}

In this paper we have developed two important contributions. First, we presented a method that uses the intersection of multiple geometric solutions in two views and in three views to determine point-to-point correspondence. Second, for each geometric model we have determined the real distance with respect to corresponding point by means of the MLESAC estimator. The main novelty of our proposal is the geometric methodology for solving the problem of the estimation of point-to-point correspondence, regardless of the angles of the points of view of the objects. We call this algorithm Bifocal Geometric Correspondence (BIGC) for the correspondence in two views.

It is important to note that the point can be occluded, but its position remains valid because our method is based on a geometric model that defines the scene. We also show that the use of multiple random solutions makes it possible to improve the performance of the correspondence in two views. Although our method starts from the basis that there is a set of points in previous correspondence necessary to determine the fundamental matrices, it is designed to maximize the correspondences in specific regions of each image.

In the experiments performed we considered outdoor images. The results obtained with these sets indicate that the BIGC algorithm was capable of determining point-to-point correspondence precisely, with a performance $\mathrm{F}$-score $=97 \%$ in stereo images at a discretized distance $\epsilon=0$ pixels for outdoor images.For all the images analyzed, we showed that the point-to-point correspondence can be generated through a multiple geometric relation between two views. In relation to this last point we stress that out method can be applied as support of industrial control to follow-up faults in uncalibrated sequences, among other applications.

Acknowledgment. This work was supported by the National Commission of Science and Technology (CONICYT, Chile). Fondecyt grant no. 11100098. 


\section{References}

1. Barron, J.L., Fleet, D.J., Beauchemin, S.S.: Performance of optical flow techniques. International Journal of Computer Vision 12(1), 43-77 (1994)

2. Bartoli, A., Sturm, P.: Nonlinear estimation of the fundamental matrix with minimal parameters. IEEE Transactions on Pattern Analysis and Machine Intelligence 26(3), 426-432 (2004)

3. Bay, H., Ess, A., Tuytelaars, T., Gool, L.: Surf: Speeded up robust features. Computer Vision and Image Understanding (CVIU) 110(3), 346-359 (2008)

4. Caspi, Y., Irani, M.: A step towards sequence-to-sequence alignment. In: IEEE Conference on Computer Vision and Pattern Recognition (CVPR), pp. 682-689. IEEE, Hilton Head Island (2000)

5. Caspi, Y., Simakov, D., Irani, M.: Feature-based sequence-to-sequence matching. International Journal of Computer Vision 68(1) (2006)

6. Dempster, A.P., Laird, N., Rubin, D.B.: Maximum likelihood from incomplete data via the em algorithm. Journal of the Royal Statistical Society, Series B 39, 1-38 (1977)

7. Fischler, M., Bolles, R.: Random sample consensus: a paradigm for model fitting with applications to image analysis and automated cartography. Communications of the ACM 24(6), 381-395 (1981)

8. Fitzgibbon, A.: Robust registration of $2 \mathrm{~d}$ and $3 \mathrm{~d}$ point sets. Image and Vision Computing 21(13-14) (December 2003)

9. Hartley, R., Zisserman, A.: Multiple View Geometry in Computer Vision. Cambridge University Press, Cambridge (2000)

10. Kadir, T., Zisserman, A., Brady, M.: An Affine Invariant Salient Region Detector. In: Pajdla, T., Matas, J. (eds.) ECCV 2004. LNCS, vol. 3021, pp. 228-241. Springer, Heidelberg (2004)

11. Lowe, D.G.: Distinctive image features from scale-invariant keypoints. Int. J. Comput. Vis. 60(2), 91-110 (2004)

12. Moreels, P., Perona, P.: Evaluation of features detectors and descriptors based on 3d objects. International Journal of Computer Vision 73(3) (2007)

13. Olson, D.L., Delen, D.: Advanced Data Mining Techniques. Springer, Heidelberg (2008)

14. Romano, R.: Projective Minimal Analysis of Camera Geometry. Phd. thesis, M.I.T., USA (May 2002)

15. Tordoff, B.J., Murray, D.W.: Guided-mlesac: faster image transform estimation by using matching priors. IEEE Transactions on Pattern Analysis and Machine Intelligence 27(10), 1523-1535 (2005)

16. Torr, P.: Bayesian model estimation and selection for epipolar geometry and generic manifold fitting. International Journal of Computer Vision 50(1), 35-61 (2002)

17. Torr, P., Zisserman, A.: Mlesac: A new robust estimator with application to estimating image geometry. Computer Vision and Image Understanding 78, 138-156 (2000)

18. Tuytelaars, T., Mikolajczyk, K.: Local invariant feature detectors: A survey. Computer Graphics and Vision 3(3), 177-280 (2007)

19. Vidal, R., Ma, Y., Soatto, S., Sastry, S.: Two-view multibody structure from motion. International Journal of Computer Vision 68(1) (June 2006)

20. Zhang, Z., Deriche, R., Faugeras, O., Luong, Q.T.: A robust technique for matching two uncalibrated images through the recovery of the unknown epipolar geometry. Artificial Intelligence 78(1-2), 87-119 (1995) 